\title{
Differential Effects of Fibromodulin Deficiency on Mouse Mandibular Bones and Teeth: A Micro-CT Time Course Study
}

\author{
Michel Goldberg ${ }^{a} \quad$ Arnaud Marchadier $^{a} \quad$ Catherine Vidal $^{a}$ b Yassine Harichane $^{a}$ \\ Agnès Kamoun-Goldrat $^{a}$ Odile Kellermann ${ }^{a}$ Tina Kiltss ${ }^{c}$ Marian Young ${ }^{c}$ \\ a UMR-S U 747 INSERM, Université Paris-Descartes, Biomédicale des Saints-Pères, and ${ }^{b}$ Pasteur Institute, Paris, France; \\ 'NIH/NIDCR, Bethesda, Md., USA
}

\section{Key Words}

Fibromodulin $\cdot$ Mandible $\cdot$ Molar $\cdot$ Micro-CT •

Mineralization

\begin{abstract}
Fibromodulin (Fmod) is a keratan sulfate small leucine-rich proteoglycan which is enriched in bones and teeth. In order to determine its functions on bone and tooth mineralization we characterized the phenotype of Fmod-deficient (Fmod$\mathrm{KO})$ mice using a new-generation microfocus computerized tomography system (micro-CT) and software allowing advanced visualization of 3-D data. Three-week-old and 10week-old Fmod-KO mandibles and teeth were compared with those of age-matched wild-type (WT) mice. In both young and mature mice the Fmod-KO mandibles were hypomineralized, especially the posterior (proximal) part of the mandible as it appeared to be the main target of the molecule deficiency whereas less extensive alterations were found in the alveolar bone. In transverse sections, larger marrow spaces were observed in the Fmod-KO mice compared with age-matched young or mature WT mice. Quantitative evaluation of the pulp volume of the first molar and 3-D reconstructions suggested that dentinogenesis was diminished in 3-week-old Fmod-KO teeth. In contrast, increased dentin formation was found in 10-week-old Fmod-
\end{abstract}

KO mice and it was accompanied by a reduced pulp volume. Thus, the differential effects of Fmod deficiency on bones and teeth appear to diverge in adult mice. This may result from the previously reported differences in the molecular weight of Fmod in the 2 tissues or from compensatory mechanisms due to the overexpression of DSP and DMP-1 in the dental pulp of Fmod-KO. It is also possible that a single molecule plays diverging roles in a tissue-specific or region-specific manner.

Copyright $\odot 2011$ S. Karger AG, Basel

\section{KARGER}

๑ 2011 S. Karger AG, Basel

Fax +4161306 1234

E-Mail karger@karger.ch

www.karger.com
Accessible online at: www.karger.com/cto

\section{Abbreviations used in this paper}

Bgn

BSP

biglycan

CS/DS

Dcn

DMP1

DSP

Fmod

KS

micro-CT microfocus computed tomography system

PGs

SIBLINGs

SLRPs

WT bone sialoprotein

chondroitin sulfate/dermatan sulfate decorin dentin matrix protein-1 dentin sialoprotein fibromodulin keratan sulphate proteoglycans small integrin-binding ligand, $\mathrm{N}$-linked glycoproteins small leucine-rich proteoglycans wild type 


\section{Introduction}

Small leucine-rich proteoglycans (SLRPs) are structural proteins of the extracellular matrix, present in mineralized tissues, also involved in intracellular signal regulation. Five distinct families of SLRPs have been identified to date [Schaefer and Iozzo, 2008]. Class I includes decorin (Dcn) and biglycan (Bgn), 2 chondroitin sulfate/ dermatan sulfate (CS/DS) proteoglycans (PGs) implicated in bone and dentin mineralization. Class II members contain primarily keratan sulfate (KS) and an undersulfated form of KS. Lumican, fibromodulin (Fmod), and osteoadherin are members of class II KS PGs. These 3 molecules have been identified in mineralized tissues [Embery et al., 2001]. In contrast, SLRPs that are members of class III to class V have not yet been detected in mineralized tissues and therefore are not considered here.

Gene-targeting studies on Dcn-, Bgn-, and Fmod-KO mice have established a link with dental and bone abnormalities and support the concept that these molecules play roles in the mineralization processes [Ameye and Young, 2002; Goldberg et al. 2005]. The roles of Fmod still need to be further deciphered. Our first approach was to map KS in dental tissues, and immunolocalization studies revealed a non-uniform distribution in predentin with a gradient, suggesting a potential role in dentin mineralization [Embery et al., 2001]. Using both light and electron immunohistochemistry on 1-day-old wild-type (WT) and Fmod-KO mice, we uncovered a potential role of this molecule in collagen fibrillogenesis [Goldberg et al., 2006]. Specifically, these studies showed that the diameter of collagen fibrils is larger in the predentin of Fmod-KO mice compared with WT mice. Additionally, these studies showed that dentin is hypomineralized and that there are structural defects in the forming dentin and enamel [Goldberg et al. 2006]. Further, enhanced immunolabeling was detected for 3 small integrin-binding ligand, N-linked glycoproteins (SIBLINGs) [Fisher and Fedarko, 2003], dentin sialoprotein (DSP), dentin matrix protein-1 (DMP1), and bone sialoprotein (BSP), suggesting the occurrence of compensatory mechanisms in dental tissues; this finding was confirmed by Western blotting [Goldberg et al., 2009].

Genetically modified mice are highly valuable models for research on bone and tooth mineralization and for a better understanding of human bone and tooth pathologies [Ameye and Young, 2002]. Normally, experimentation to evaluate dental and skeletal phenotypes involves destructive and time-consuming procedures. To improve this process and, at the same time, reliably quantitate the mineralization process, we used a new-generation microfocus computed tomography system (micro-CT) (Viscom, X8060 NDT). The Viscom system provides an optimal compromise between spatial resolution, acquisition time, and high-quality imaging. The dedicated software (VoxBox; UsefulProgress) allows advanced visualization of 3-D data with ray-casting algorithms. Micro-CT imaging is quicker and provides high-resolution qualitative and phenotypic analysis. Consequently, we investigated the effects of Fmod deficiency on mouse mandibles and obtained: (i) an accurate qualitative and quantitative analysis of the mandibular bone, (ii) a 3-D reconstruction of the pulp of the molar (a new approach for the quantitative evaluation of dentinogenesis), and (iii) a time-course study which was carried out in order to determine whether the effects of the gene targeting are permanent or, rather, transient. Therefore, teeth and mandibles from 3- and 10-week-old Fmod-KO mice were compared with agematched WT mice.

\section{Materials and Methods}

All experiments were performed under an institutionally approved protocol for the use of animals in research (No. NIDCRIRP-09-536). Fmod-KO mice were generated by gene targeting in embryonic stem cells. All mice were genotyped by PCR analysis using DNA isolated from a small tail biopsy as previously described. Data obtained from mandibles of 3- and 10-week-old Fmod-KO mice were compared with age-matched WT mice (16 mice/group).

\section{Micro-CT Imaging}

To improve this process and at the same time reliably quantitate the mineralization process, for high-resolution 3-D imaging of mandibles we used a new-generation micro-CT originally devoted to industrial applications (Viscom, X8060 NDT). The Viscom system provides an optimal compromise between spatial resolution, acquisition time, and high-quality imaging. The dedicated software (VoxBox; UsefulProgress) allows advanced visualization of 3-D data with ray-casting algorithms. X-ray source parameters were: $60 \mathrm{kV}, 200 \mu \mathrm{A}$, and an exposure time of $1 \mathrm{~s}$ for each frame. Mandible 3-D images had an isotropic resolution of $13 \mu \mathrm{m}$ and a dynamic range of 16 bits.

\section{Calibration of Grey Levels}

Contrasts in grey levels of CT images relied on differential attenuations of X-rays through the material. Theoretically, a monochromatic X-ray beam will give a direct relation between grey level and density. Because the micro-CT X-ray source is polychromatic, it could give rise to artifactual variations of the grey scale. Therefore, in order to obtain an accurate comparison between the mandibles of the 2 groups of mice, we performed a systematic calibration of the CT images. Two references were used: (i) the 
Fig. 1. Micro-CT imaging of the mandible clearly shows the 3 different domains with an unequally distributed pattern of thickness and mineralization.

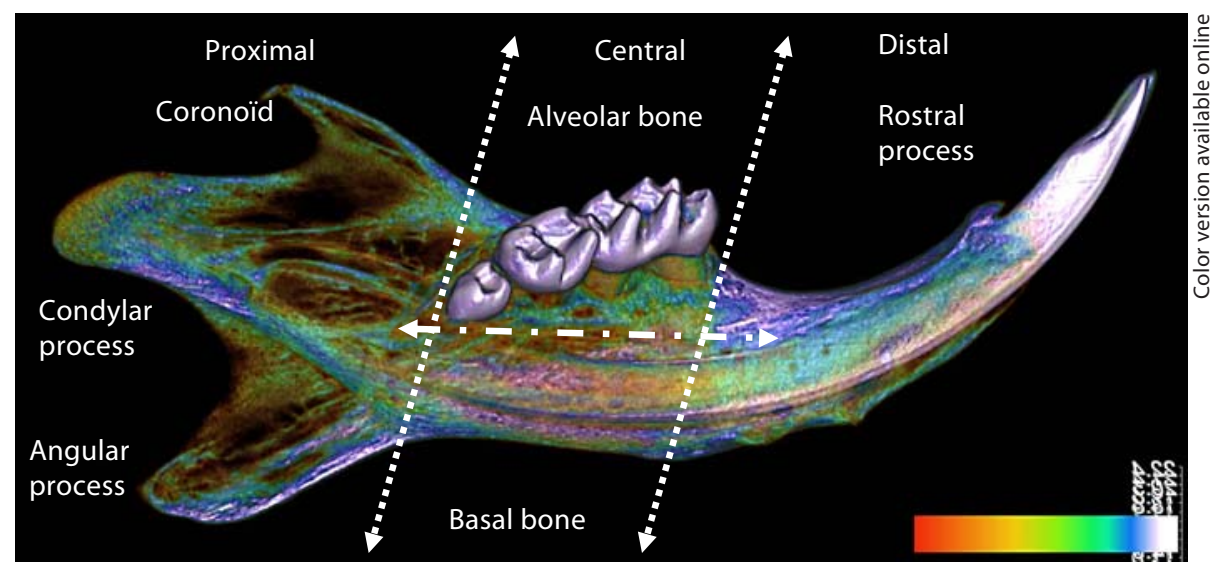

enamel at the tip of the incisor, the density of which is constant in all mice, and (ii) the background grey level. Each mandible histogram was checked for its grey scale level and shifted if necessary according to the defined references before proceeding to the 3-D reconstruction.

Visualization with Volume Rendering

We used VoxBox software (UsefulProgress) for the advanced visualization of 3-D datasets with ray-casting algorithms. Comparison between control and mutant mouse mandibles was made by applying the same transfer function for each 3-D data set, thus allowing the detection of shape, color, and density discrepancies. The same protocol was used to visualize $2-\mathrm{D}$ virtual slices across the mandible.

Dental Pulp Volume and 3-D Reconstruction

The dental pulp of the molar refers to the inner most portion of the tooth that contains the crown and root pulp (also including in the calculation a very thin predentin layer). It constitutes a 3-D closed region and we used the connected threshold image filter available in the Insight Toolkit to extract the 'pulp' volume.

\section{Results}

Different Domains of the Mandible and Identification of the Target of Fmod Deficiency

The mouse mandible has long served as a model system for complex morphological structures. According to Klingenberg et al. [2004], the mandible consists of 2 main modules, i.e. the alveolar region and the ascending ramus. This appears to be an oversimplification, and the images of the mouse mandible obtained by micro-CT followed by 3-D advanced visualization using VoxBox software showed a more complex organization. Three distinct parts formed the mandible. The distal or rostral (anterior) part differed from the central part and represents the most mineralized module. The central part was sub- divided into an upper zone containing the 3 molars implanted in an alveolar bone, whereas in the lower part the basal bone encircled the incisor. The posterior (proximal) part included endochondral-derived domains: the angular, condylar, and coronoid processes. The dark brown color indicates that this area is thinner and consequently reflects less mineralization than the rest of the mandible (fig. 1).

The main target of Fmod deficiency was the posterior (proximal) part of the mandible, including condylar, coronoid, and angular processes, which was less dense in 3-week-old Fmod-KO mice compared with the WT. This was also detectable in the alveolar bone (upper middle) but became undetectable in the basal and rostral parts of the mandible (fig. 2a, b). The difference between Fmod$\mathrm{KO}$ and the WT was reduced but still detected in the 10-week-old mice (fig. 2c, d).

Altogether, these findings indicate that (i) the mandible is a mosaic formed by different domains with an unequally distributed thickness and mineralization pattern and (ii) the proximal and alveolar parts of the mandible constitute the specific targets of Fmod deficiency.

\section{Effects of Fmod Deficiency on the Medulla}

In transverse sections of the mouse mandible virtually sliced exactly between the 1st and 2nd mandibular molars, the marrow spaces of the medulla (trabecular zone) were larger in the Fmod-KO mice compared with the WT. This broadening was observed both in the young (3-week-old) (fig. 3a, b) and in the mature (10-week-old) mice (fig. 3c, d). No difference was noted for the cortices. Therefore, bone hypomineralization was associated with the enlargement of endosteous spaces. There was no spontaneous repair or rescue of this phenotype during the aging process, at least for the period of time studied. 
Fig. 2. a Three-week-old WT mice mandibles. b Three-week-old Fmod-KO mice mandibles. The target of the deficiency is clearly identified in the proximal and central alveolar bone (white asterisks), whereas the mandible is less affected by the deficiency in 10-week-old WT (c) and Fmod$\mathrm{KO}$ (d) mice.
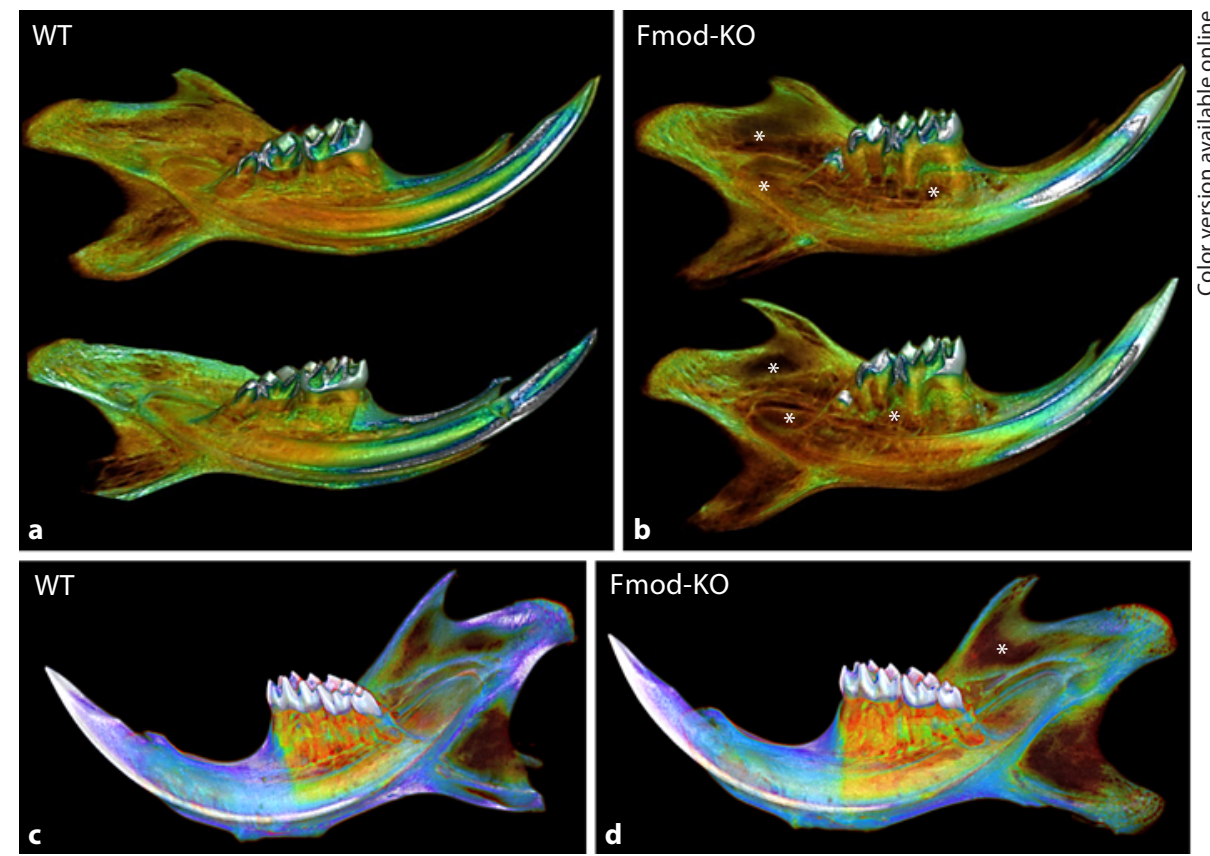
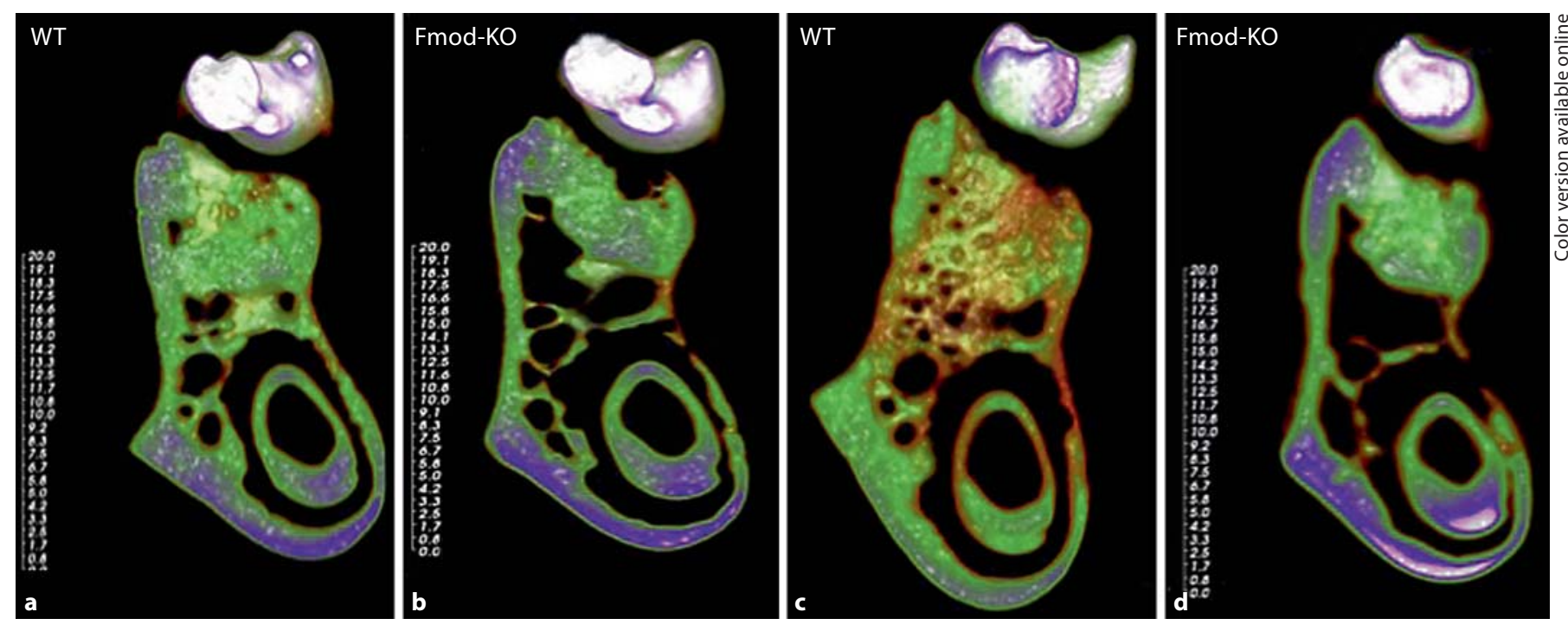

Fig. 3. Compared with 3 -week-old mice WT mice (a) and 10-week-old WT mice (c), bone marrow spaces (in black) are larger in the medulla of 3-week-old (b) and 10-week-old Fmod-KO (d) mice.

\section{3-D Reconstruction of the Pulp of the First}

\section{Mandibular Molar}

In 3-week-old Fmod-KO mice, 3-D reconstructions of the pulp showed there was reduced dentinogenesis because the volume of the pulp was larger, i.e. in the lower part of the root pulp (apical part). The length of the roots was nearly identical in the Fmod KO and WT mice (fig. $4 \mathrm{a}, \mathrm{b}, \mathrm{d}$ ). In contrast, in the 10 -week-old mice, the length of the roots was shorter in the FmodKO group and the pulp volume was restricted, suggesting a reactivation of dentinogenesis at that time (fig. 4c). 
Fig. 4. The 3-D reconstruction of the dental pulps (including the pulp and predentin) shows a larger volume in the 3-weekold Fmod-KO mice (reduced dentinogenesis) compared with age-matched WT (a, $\mathbf{b}, \mathbf{d})$, but there is a smaller pulp volume in the 1st molar of 10-week-old Fmod-KO mice (reactivated dentinogenesis) compared with age-matched WT (c). The 3-D reconstruction of the 1st mandibular molar pulp of Fmod-KO is in purple and it is in green for the WT. There was about a $17 \%$ enlargement of the pulp volume (in $\mathrm{mm}^{3}$ ) in the Fmod-KO mice.
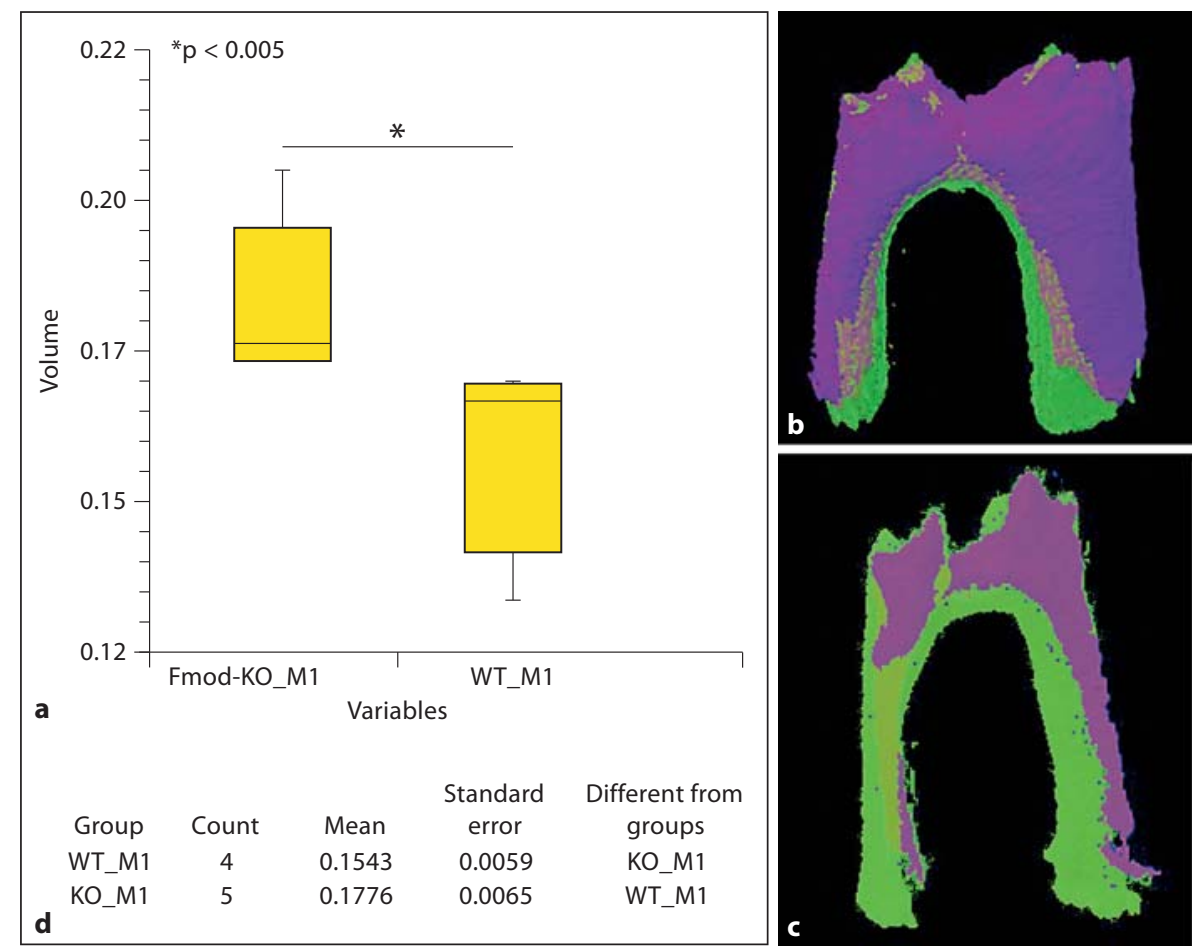

\section{Discussion}

For years there has been a debate about the functions of GAGs or PGs with regard to whether they are mineralization inhibitors or promoters; because GAGs are more abundant in osteoid and predentin, but are decreased in bone or in dentin, PGs were considered to be inhibitors that had to be enzymatically degraded before the onset of mineralization [Baylink et al., 1972; Hall et al., 1999; Embery et al. 2001]. Other reports established that CS/DS and KS PGs are present in mineralized tissues [Embery et al., 2001]. Radioautographic investigation first showed the occurrence of a dual incorporation of labeled PG precursors in dental tissues [Lormée et al., 1996]. One group of PGs is first secreted in the predentin and contributes to collagen fibrillogenesis. They are subsequently removed after enzymatic degradation. A second group of SLRPs secreted near the predentin-dentin junction is implicated in the mineralization processes and is further incorporated in the dentin as stable matrix components in the mineralized dentin. Previous studies on Bgn-, Dcn-, and Fmod-KO mice have implicated all of these molecules in dentin mineralization [Goldberg et al., 2005, 2006, 2009]. The present study confirms that Fmod influences the mineralization of bone and dental tissue.
This regulation may be due to the effect of SLRPs on collagen fibrillogenesis evidenced by the observation that larger diameters of collagen fibrils were present in the predentin of Bgn-KO and Fmod-KO teeth [Goldberg et al., 2005, 2006]. Despite some discrepancies in different reports related to the occurrence of thinner or larger diameters, depending on the site and organ studied, the influence of SLRPs on collagen cross-linking patterns during fibril growth is now well recognized [Kalamajski and Oldberg, 2010].

In this context, it is worth noting that Fmod-deficiency influences bone mineralization in both young and mature mice, whereas such effects become undetectable in dental tissues of 10-week-old Fmod-KO mice. This phenotypic change and diverging effect(s) of Fmod may be due to differences in the molecular weight of Fmod between the 2 tissues (in bone there is a single band at 52 $\mathrm{kDa}$, whereas in teeth 2 bands are detected, i.e. one at 52 $\mathrm{kDa}$ and another at $40 \mathrm{kDa}$ ), related or not to the phosphorylation or glycosylation of the molecule, and/or to compensatory mechanisms that interfere with the mineralization process [Goldberg et al., 2009].

The micro-CT method used here allows, for the first time, mapping of the different domains of the mouse mandible. In this regard, it is important to note that the 
rostral and basal parts of the mandible result from membranous ossification whereas the posterior part and alveolar bone derive from endochondral ossification. The parallel cross talk between the different domains, their level of mineralization, and the recognition of the territory influenced by Fmod imply that the loss of this PG can affect the chronologic activation of a series of specific genes, growth factors, and transcription factors which then regulate the formation of the mouse mandible.

It is now recognized that Fmod is critical in organizing niches of stem/progenitor cells [Bi et al., 2007]. In this context, the large marrow spaces seen in the medulla of the Fmod-KO mice may have a consequence in a reduc- tion of the number of bone trabecula and consequently of endosteal progenitors. This may contribute to reducing the conversion of preosteoblastic cells into osteoblastic cells and finally to a reduction of new bone formation leading to the osteoporosis induced by Fmod deficiency [Ameye and Young, 2002].

\section{Acknowledgements}

This research was supported by the IRP Program of the NIDCR/NIH and by the Fondation de l'Avenir, Etude No. ETO576.

\section{References}

Ameye, L., M.F. Young (2002) Mice deficient in small leucine-rich proteoglycans: novel in vivo models for osteoporosis, osteoarthritis, Ehlers-Danlos syndrome, muscular dystrophy, and corneal diseases. Glycobiology 12: 107R-116R.

Baylink, D., J. Wergedal, E. Thompson (1972) Loss of protein-polysaccharides at sites where bone mineralization is initiated. J Histochem Cytochem 20: 279-292.

Bi, Y., D. Ehirchiou, T.M. Kilts, C.A. Inkson, M.C. Embree, W. Sonoyama, L. Li, A.I. Leet, B.-M. Seo, L. Zhang, S. Shi, M.F. Young (2007) Identification of tendon stem/progenitor cells and the role of the extracellular matrix in their niche. Nat Med 13: 12191227.

Embery, G., R. Hall, R. Waddington, D. Septier, M. Goldberg (2001) Proteoglycans in dentinogenesis. Crit Rev Oral Biol Med 12: 331-349.

Fisher, L.W., N.S. Fedarko (2003) Six genes expressed in bone and teeth encode the current members of the SIBLING family of proteins. Connect Tissue Res 44(suppl 1): 33-40.
Goldberg, M., M. Ono, D. Septier, M. Bonnefoix, T.M. Kilts, Y. Bi, M. Embree, L. Ameye, M.F. Young (2009) Fibromodulin-deficient mice reveal dual functions for fibromodulin in regulating dental tissue and alveolar bone formation. Cells Tissues Organs 189: 198202.

Goldberg, M., D. Septier, A. Oldberg, M.F. Young, L.G. Ameye (2006) Fibromodulindeficient mice display impaired collagen fibrillogenesis in predentin as well as altered dentin mineralization and enamel formation. J Histochem Cytochem 54: 525-537.

Goldberg, M., D. Septier, O. Rapoport, R.V. Iozzo, M.F. Young, L.G. Ameye (2005) Targeted disruption of two small leucine-rich proteoglycans, biglycan and decorin, excerpts divergent effects on enamel and dentin formation. Calcif Tissue Int 77: 297-310.
Hall, R., D. Septier, G. Embery, M. Goldberg (1999) Stromelysin-1 (MMP-3) in forming enamel and predentine in rat incisor - coordinated distribution with proteoglycans suggests a functional role. Histochem J 31: 761770.

Kalamajski, S., A. Oldberg (2010) The role of small leucine-rich proteoglycans in collagen fibrillogenesis. Matrix Biol 29: 248-53.

Klingenberg, C.P., L.J. Leamy, J.M. Cheverudc (2004) Integration and modularity of quantitative trait locus effects on geometric shape in the mouse mandible. Genetics 166: 19091921.

Lormée, P., S. Lécolle, C. Beaudin, D. Septier, M. Goldberg (1996) Dual incorporation of (35S) sulfate into dentin proteoglycans acting as mineralization promotors in rat molars, and predentin proteoglycans. Calcif Tissue Int 58: 368-375.

-Schaefer, L., R.V. Iozzo (2008) Biological functions of the small leucine-rich proteoglycans: from genetics to signal transduction. J Biol Chem 283: 21305-21309. 